\title{
VALIDAÇÃO DE MÉTODO POR MEFS-HS/CG-EM PARA DETERMINAÇÃO DE CARBARIL EM PLASMA SANGUÍNEO
}

\author{
Francisco Thiago C. de Souza ${ }^{a}$, André Luiz M. Cameloa, Ricardo de Araújo Marques ${ }^{\mathrm{b}}$, Luiz Ivando Pires F. Filho ${ }^{\mathrm{c}}$, Ronald \\ F. Pinheiro ${ }^{c}$, Elisane Longhinottia ${ }^{a}$ Helena Becker ${ }^{a, *}$ \\ aDepartamento de Química Analítica e Físico-Química, Universidade Federal do Ceará, 60455-760 Fortaleza - CE, Brasil \\ 'Departamento de Química Orgânica e Inorgânica, Universidade Federal do Ceará, 60455-760 Fortaleza - CE, Brasil \\ 'Departamento de Medicina Clínica, Universidade Federal do Ceará, 60430-160 Fortaleza - CE, Brasil
}

Recebido em 09/09/2016; aceito em 16/01/2017; publicado na web em 09/03/2017

\begin{abstract}
METHOD VALIDATION FOR HS-SPME / GC-MS FOR DETERMINATION OF CARBARYL IN BLOOD PLASMA. The aim this research is to develop a HS-SPME/GC-MS method to analyze the carbaryl in blood plasma samples of the rural workers, who live round the perimeter of Chapada do Apodi-CE. This was achieved by chromatographic signal of the 1-naftol, degradation product of carbaryl with the intention of evaluating the degree of exposure of the workers to pesticides, causing chromosome abnormalities as already evidence in appropriate literature. Three different types of covered SPME fibers were tested and a full factorial design with central point in two levels. The variables optimized were temperature and ionic strength and temperature was the response in the design. Study was undertaken to determinate the equilibrium time. The thermal degradation of carbaryl in SPME headspace was analyzed by ${ }^{1} \mathrm{H}-\mathrm{NMR}$. Proposed method achieved low limits of detection and quantification (1.5 and $5 \mathrm{ng} \mathrm{mL}{ }^{-1}$, respectively) with good precision and accuracy, using the method of weighted least squares. However, the proposed method can be considered a good alternative way of monitoring carbaryl in blood plasma by analytical signal of 1-naftol. None of the plasma analyzed presented a concentration of 1-naftol compound higher than limit of detection of the method proposed here.
\end{abstract}

Keywords: carbaryl; blood plasma; HS-SPME, GC-MS

\section{INTRODUÇÃO}

Segundo o Relatório Global sobre Desenvolvimento e Água 2015, das Organizações das Nações Unidas (ONU), a tendência para 2030 é que a população global, em pleno crescimento, necessite de $35 \%$ a mais de alimento, $50 \%$ a mais de energia e conviva com um déficit de $40 \%$ de água. ${ }^{1}$ Esta previsão chama a atenção no que concerne a qualidade dos produtos oferecidos para atender essa crescente demanda. Dentre estas preocupações pode-se destacar o uso de agrotóxicos na produção de alimentos. Somente o mercado brasileiro de agrotóxicos totalizou US\$12,249 bilhões em 2014, contra US $\$ 11,454$ bilhões em 2013, representando aumento de $6,9 \%$ em apenas 12 meses. $^{2}$

A presença de agrotóxicos em alimentos e no meio ambiente vem sendo mostrada em estudos realizados nessa área, relatando a presença em quantidades traços desses compostos ${ }^{3-6}$ Resultados de uma análise de águas da região da chapada do Apodi-CE, divulgado pelo dossiê $\mathrm{ABRASCO},{ }^{7}$ destaca que o carbaril, um pesticida da classe química dos carbamatos, foi detectado em todas as amostras de água analisadas.

Os carbamatos vêm substituindo, com frequência, os compostos organofosforados e, principalmente, os organoclorados nas plantações devido sua baixa persistência nos meios ambientais e biológicos. ${ }^{8,9} \mathrm{~A}$ baixa persistência dessa classe de agrotóxicos se deve à instabilidade térmica, hidrólise ocorrida em pHs básicos (Figura 1) ou facilidade em sofrer oxidações. ${ }^{10,11}$

No entanto, na literatura especializada são encontrados vários trabalhos que reportam o efeito toxicológico do carbaril em ratos, como a inibição da AchE; ${ }^{12,13}$ alterações em marcadores de estresse oxidativo e parâmetros bioquímicos séricos: ${ }^{14}$ prejuízos à respiração celular e toxicidade mitocondrial quando presente em elevadas

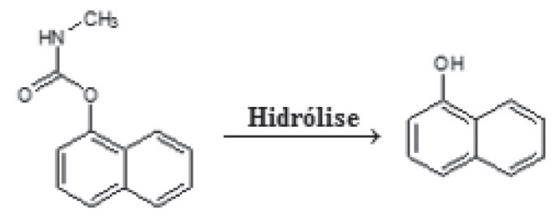

Figura 1. Hidrólise do carbaril em seu respectivo correspondente fenólico 1-naftol

concentrações, ${ }^{15}$ além de contribuir para o desenvolvimento de alergia, doenças auto-imunes, câncer ou infecções, facilitada pelo desequilíbrio da resposta imune Th1/Th2. ${ }^{16}$

Sua toxicidade também é confirmada em peixes, ${ }^{17,18} \mathrm{em}$ girinos, atuando como um perturbador endócrino e afetando as vias biológicas associadas aos hormônios da tireóide ${ }^{19}$ e nas espécies Folsomia candida, Eisenia andrei, Triticum aestivum e Brassica rapa observando-se o aumento dessa toxicidade com a variação da temperatura. ${ }^{20}$ Esses efeitos observados têm gerado uma preocupação na utilização do carbaril, embora o mesmo seja rapidamente metabolizado e excretado pelas espécies intoxicadas.

Nesse contexto é de fundamental importância que novas metodologias de análises sejam desenvolvidas para o monitoramento desse e de outros pesticidas em amostras biológicas de seres humanos que se expõem de maneira continua a esses compostos. ${ }^{21-25}$

Esse trabalho teve como objetivo analisar carbaril em amostras de plasma sanguíneo de trabalhadores rurais do perímetro irrigado da Chapada do Apodi-CE, pelo sinal cromatográfico do produto de degradação térmica 1-naftol, com o intuito de avaliar o grau de exposição ao qual esses trabalhadores estão sujeitos, associando à possível detecção desses agrotóxicos a anormalidades cromossômicas já evidenciadas na literatura. ${ }^{26,27}$ 


\section{PARTE EXPERIMENTAL}

\section{Reagentes e soluções}

Carbaril (Sigma-Aldrich ${ }^{\circledR}$-99,8\%) e pirimicarbe (Sigma-Aldrich ${ }^{\circledR}$ -98,5\%) utilizado como padrão substituto (PS) ou "padrão surrogate", metanol (Sigma-Aldrich ${ }^{\circledR}$-99,9\%), acetona (Sigma-Aldrich ${ }^{\circledR}-99,9 \%$ ), hidrogêno fosfato de sódio $\mathrm{Na}_{2} \mathrm{HPO}_{4}\left(\mathrm{Vetec}^{\circledR}-99,0 \%\right.$ ), di-hidrogêno fosfato de sódio $\mathrm{KH}_{2} \mathrm{PO}_{4}\left(\right.$ Vetec $\left.^{\circledR}-98,0 \%\right)$ e cloreto de sódio $\mathrm{NaCl}$ $\left(\right.$ Vetec $\left.^{\circledR}-99,0 \%\right)$ foram usados. A água utilizada foi obtida através de um sistema de purificação de água Milli-Q (Millipore ${ }^{\circledR}$ ).

Soluções padrão estoque de carbaril e pirimicarbe (PS) $10 \mathrm{mg}$ $\mathrm{L}^{-1}$ foram preparadas separadamente em metanol e armazenadas a $-20{ }^{\circ} \mathrm{C}$. As demais soluções padrões foram obtidas por diluição da solução padrão estoque, com metanol, no momento da análise. A solução tampão Sorensen $\mathrm{pH}$ 5,5 foi preparada pela mistura de 25 $\mathrm{mL}$ de $\mathrm{Na}_{2} \mathrm{HPO}_{4}$ 0,084 mol L-1 e $475 \mathrm{~mL}$ de $\mathrm{KH}_{2} \mathrm{PO}_{4} 0,067 \mathrm{~mol} \mathrm{~L}^{-1}$ e armazenadas a $4{ }^{\circ} \mathrm{C}$.

\section{Condições do CG-EM}

As análises cromatográficas foram realizadas utilizando um cromatógrafo gasoso (Shimadzu GC-2010), equipado com detector seletivo de massas (Shimadzu GCMS QP-2010), com coluna capilar de sílica fundida DB-5 (20 m x 0,18 mm d.i. x 0,4 $\mu \mathrm{m})$. As condições experimentais foram: temperatura do injetor, interface e fonte de íons de 260,270 e $260{ }^{\circ} \mathrm{C}$, respectivamente, gás de arraste hélio com velocidade linear do método determinada experimentalmente, para garantir a melhor eficiência da coluna e injetor no modo splitless. A programação de temperatura do forno foi iniciada com $80^{\circ} \mathrm{C} \mathrm{du}-$ rante 1 minuto e elevada a $165^{\circ} \mathrm{C}$ a $20^{\circ} \mathrm{C} / \mathrm{min}$, subsequentemente elevou-se a $225^{\circ} \mathrm{C}$ a $10{ }^{\circ} \mathrm{C} / \mathrm{min}$, finalmente, a $290{ }^{\circ} \mathrm{C}$ a $35^{\circ} \mathrm{C} / \mathrm{min}$, sendo a temperatura final mantida por um minuto. $\mathrm{O}$ espectrômetro de massas foi operado no modo íon-positivo de impacto de elétrons (IE) a $70 \mathrm{eV}$. Os fragmentos foram analisados entre $40-450 \mathrm{~m} / \mathrm{z}$. $\mathrm{Na}$ determinação semi-quantitativa MIS (monitoramento de íons selecionados), utilizou-se um único fragmento para cada composto, carbaril (1-naftol) $144 \mathrm{~m} / \mathrm{z}$ e pirimicarbe $166 \mathrm{~m} / \mathrm{z}$, sendo o segundo utilizado como padrão substituto.

\section{Condições da MEFS-Headspace e preparo da amostra de plasma}

Foram avaliados três tipos diferentes de revestimentos comerciais disponíveis (PDMS/DVB - $65 \mu \mathrm{m}, \mathrm{PA}-85 \mu \mathrm{m}$ e CAR/PDMS - 75 $\mu \mathrm{m})$ obtidos na Supelco ${ }^{\circledR}$. Um planejamento experimental do tipo $2^{2}$ com componente central foi realizado com as variáveis temperatura e força iônica $(\mathrm{NaCl})$. Quando necessário foi utilizado uma expansão do planejamento, para planejamento em estrela com adição dos níveis $\pm \sqrt{ } 2$ (planejamento quadrático), conforme a Tabela 1. As fibras foram condicionadas no injetor do CG com temperatura e tempo recomendado pelo fabricante. Após esse condicionamento as fibras eram submetidas a uma limpeza no injetor do CG a uma temperatura de $260{ }^{\circ} \mathrm{C}$ durante $10 \mathrm{~min}$, sempre e antes de qualquer extração.

O frasco (vial $40 \mathrm{~mL}$ ) foi colocado em um sistema sob agitação magnética constante de 1000 rpm e o pH 5,5 foi ajustado com solução tampão. A fibra foi introduzida através do septo e mantida no espaço superior do vial (modo Headspace) por um tempo de extração fixo de 20 minutos, posteriormente, retirada e imediatamente inserida no sistema de injeção de CG durante 7 minutos para a dessorção dos analitos. Após determinada às melhores condições do planejamento, um estudo da variação do tempo de extração (5 - 180 minutos) foi realizado, para que o tempo de equilíbrio fosse determinado.
Tabela 1. Variáveis utilizadas no planejamento experimental $2^{2}$ com componente central do tipo estrela

\begin{tabular}{lccccc}
\hline \multirow{2}{*}{ Fatores } & \multicolumn{5}{c}{ Níveis } \\
\cline { 2 - 6 } & $-\sqrt{ } 2$ & $(-1)$ & 0 & $(+1)$ & $\sqrt{ } 2$ \\
\hline Temperatura (T) ${ }^{\circ} \mathrm{C}$ & 40 & 50 & 75 & 100 & 110 \\
Força Iônica (FI) \% p/v & 0,9 & 5 & 15 & 25 & 29,5 \\
\hline
\end{tabular}

Para o preparo das amostras de plasma sanguíneo, cedidas pelo departamento de Medicina Clínica da UFC, uma alíquota de $500 \mu \mathrm{l}$ foi transferida para um eppendorf de $1,5 \mathrm{~mL}$, sendo adicionados 50 $\mu \mathrm{L}$ de metanol e $650 \mu \mathrm{L}$ de acetona para precipitação das proteínas e essa solução foi agitada em vortex por 30 segundos. A amostra foi centrifugada por 12 minutos a $4000 \mathrm{rpm}$, o sobrenadante foi transferido para o vial e concentrado a $500 \mu \mathrm{L}$ com fluxo de gás nitrogênio. Em seguida $0,875 \mathrm{~g}$ de $\mathrm{NaCl}$ e 2,5 mL de solução tampão Sorensen $\mathrm{pH}$ 5,5 foram adicionados e o vial imediatamente tampado com um septo PTFE.

\section{Condições do RMN $\mathbf{H}^{\mathbf{1}}$}

Para determinação dos deslocamentos químicos $(\delta)$ para os sinais de H, dos padrões de carbaril e 1-naftol foi utilizado o sistema RMN, Brucker, modelo Avance DRX-300 e modelo Avance DRX-500. Para a obtenção dos espectros, $10 \mathrm{mg}$ de amostra padrão de carbaril foi dissolvida em $100 \mu \mathrm{L}$ metanol em vial de $40 \mathrm{~mL}$ e submetida às condições de extração otimizadas no planejamento fatorial; a amostra foi então liofilizada, e foram adicionados $10 \mathrm{~mL}$ de metanol para separar os analitos dos sais inorgânicos. Essa solução foi levada à secura sob fluxo de $\mathrm{N}_{2}$ e, em seguida, foram adicionados $600 \mu \mathrm{L}$ de clorofórmio deuterado. A partir daí deu-se a análise da solução por RMN. O espectro obtido foi confrontado com os espectros individuais dos padrões para determinação da \% de 1-naftol produzido pela degradação térmica do carbaril nas condições de trabalho.

\section{Validação do método}

O método foi validado segundo as orientações ANVISA. ${ }^{28,29}$ Embora as mesmas não sejam específicas para análise de pesticidas em amostras biológicas, são as que melhor orientam a validação de metodologia bioanalítica no Brasil.

\section{Seletividade}

A seletividade do método foi avaliada em amostras de plasma obtidas de seis indivíduos, sendo 4 amostras normais, uma lipêmica (amostra com alto teor de lipídeos) e uma hemolisada (amostra biológica contendo hemácias lisadas em grau pré-definido e especificado pelo laboratório bioanalítico).

\section{Efeito residual}

Foram realizadas três injeções da mesma amostra branco, sendo uma antes e duas logo após a injeção de uma amostra processada do Limite Superior de Quantificação (LSQ). Esses resultados foram comparados com aqueles obtidos das amostras processadas do Limite Inferior de Quantificação (LIQ), sendo esses limites de quantificação a maior e a menor concentração da curva de calibração, respectivamente.

\section{Linearidade, Limite de Detecção (LD) e Limite de Quantificação (LQ)}

A curva analítica foi determinada pelo método de superposição da matriz (matrix-matched), em que quantidades conhecidas do 
agrotóxico foram adicionadas à amostra de plasma branco de um mesmo paciente saudável, nas seguintes concentrações 12,0; 30,0;

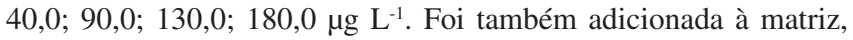
antes do processo de MEFS-HS, concentração conhecida do (PS). Esse padrão é um composto quimicamente diferente da substância de interesse, mas representativo de seu comportamento, com o intuito de corrigir pequenas mudanças em variáveis experimentais (extração e análise). Algumas vezes este composto é denominado padrão interno. ${ }^{30} \mathrm{~A}$ resposta analítica foi determinada através da razão entre as áreas analito/(PS), obtidas no cromatograma.

A linearidade e o modelo matemático "equação da reta" foram determinados pelo Método dos Mínimos Quadrados Ordinários (MMQO), e devido às análises estatísticas de falta de ajuste, através da tabela de teste $\mathrm{F}$ de variância ${ }^{31}$ (ANOVA) e heterocedasticidade, através da análise dos resíduos, ${ }^{32,33}$ assim como utilizando os testes de Cochran e F de Hartley, ${ }^{33}$ fez-se necessária uma expansão do modelo, para o Método dos Mínimos Quadrados Ponderados (MMQP), adotando-se como peso $\left(w_{i}\right)$, com o intuito de corrigir variações nos resíduos "heterocedasticidade" e tornar o modelo mais realístico e adequado ao conjunto de dados, ${ }^{32}$ sendo esses tratamentos estatísticos melhor descritos em Souza et al. (não publicado). ${ }^{34}$

$$
w_{i}=\frac{s_{i}^{-2}}{\sum_{i} s_{i}^{-2} / n}
$$

Sendo, $s_{i}^{-2}$ o inverso da variância, e $n$ é o número de pontos da curva $(n=6)$

O LD foi determinado como sendo três vezes os desvios padrão do intercepto $\left(\mathrm{b}_{w i}\right)$ dividido pelo coeficiente angular de três curvas e o LQ determinado como sendo dez vezes o desvio padrão do intercepto, dividido pelo coeficiente angular de três curvas.

\section{Precisão e exatidão}

A precisão e exatidão do método foram avaliadas em dois níveis (intra e intercorridas), feitas em amostras de plasma de pacientes saudáveis; o plasma foi dopado em níveis de concentração, baixo LIQ, médio CM e alto LSQ, com replicatas de cada ponto $(n=5)$, sendo a precisão determinada em função do desvio padrão relativo da concentração obtida na linearidade e a exatidão determinada em função da recuperação da concentração média experimental e a concentração média estimada pela linearidade do método.

\section{Aplicação do método em amostras de pacientes voluntários}

Amostras de sangue de 10 trabalhadores da região de Limoeiro do Norte-CE, cenário de intensos conflitos devido ao número elevado de casos de câncer e ao elevado uso de agrotóxicos, foram coletadas por meio de doação voluntária, selecionadas de acordo com o histórico de vida dos pacientes de diferentes faixas etárias e sexo; as amostras foram processadas para separação do plasma sanguíneo, pelo grupo de Citogenômica do Câncer, do Departamento de Medicina da Universidade Federal do Ceará, e então analisadas pelo método aqui desenvolvido.

Esse estudo foi desenvolvido em parceria com a pesquisa: Estudo das alterações citogenômicas da medula óssea de trabalhadores rurais expostos a agrotóxicos, ${ }^{27}$ tendo aprovação pelo Comitê de Ética em Pesquisa do Hospital Universitário Walter Cantídio da Universidade Federal do Ceará, através do Protocolo CEP N ${ }^{\circ}$ 016.02.11, cumprindo todas as exigências da resolução 196/96 do Conselho Nacional de Saúde.

\section{RESULTADOS E DISCUSSÃO}

\section{Otimização do método para quantificação do carbaril em CG- EM}

Com base na característica de instabilidade térmica do carbaril, já bem estabelecida na literatura, ${ }^{8,9,35,36}$ a quantificação desenvolvida nesse trabalho foi realizada pelo monitoramento do sinal do seu único produto de degradação térmica (Figura 2a), indicado pelo banco de dados de espectros de massa do software do equipamento CG-EM e confirmado pela injeção de seu padrão como sendo o 1-naftol (Figura 2b).

Pela inspeção do cromatograma apresentado na Figura 2a é possível observar claramente o perfil de degradação térmica do carbaril, sendo o mesmo quase que totalmente convertido em 1-naftol (94\%)

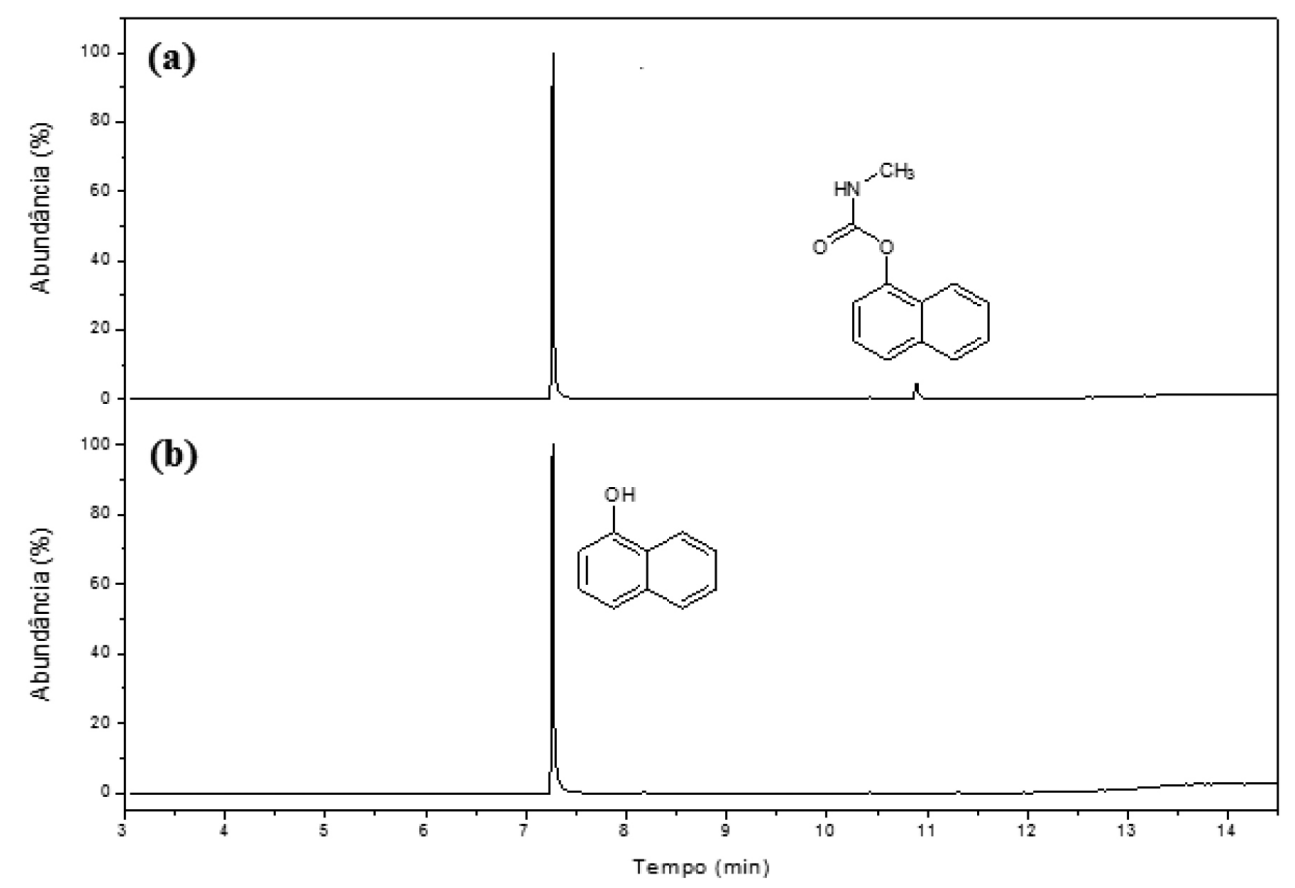

Figura 2. Cromatograma do padrão carbaril (a), e do padrão 1-naftol (b) 
quando exposto às condições de alta temperatura do injetor $\left(260^{\circ} \mathrm{C}\right)$ que, associadas à presença dos grupos silanóis do liner, potencializam o processo de degradação térmica do analito. ${ }^{37} \mathrm{Na}$ Figura $2 \mathrm{~b}$ é possível observar que o composto 1-naftol é estável termicamente, o que possibilita sua utilização como composto de referência na quantificação do carbaril.

\section{Condições MEFS-headspace por planejamento experimental $2^{2}$ utilizando componente central designer}

Existem muitos fatores que influenciam a eficiência de extração dos analitos, como o revestimento de fibra de MEFS, temperatura de extração, tempo de extração, força iônica e agitação, dentre outros. ${ }^{38}$ Uma efetiva agitação é necessária em análises de amostra de sangue, devido à elevada viscosidade da amostra e inferior coeficiente de difusão do analito. ${ }^{39}$

A avaliação da análise de variância para o modelo linear mostrou-se insatisfatória, sendo então necessária a expansão para um modelo quadrático. Assim, foi realizado em um segundo bloco (dias diferentes) e a análise de variância (Tabela 2) mostrou que esse é o modelo mais adequado e que melhor representa a superfície de resposta estudada.

Tabela 2. Análise de variância para o ajuste do modelo quadrático $y=b_{0}+b_{1} \cdot x_{1}$ $+b_{2} \cdot x_{2}+b_{11} \cdot\left(x_{1}\right)^{2}+b_{22} \cdot\left(x_{2}\right)^{2}+b_{12} \cdot x_{1} \cdot x_{2}$, realizados em dois blocos para o carbari

\begin{tabular}{lccc}
\hline $\begin{array}{l}\text { Fonte de } \\
\text { variação }\end{array}$ & $\begin{array}{c}\text { Soma } \\
\text { quadrática }\end{array}$ & $\begin{array}{c}\mathrm{N}^{\circ} \text { graus de } \\
\text { liberdade }\end{array}$ & $\begin{array}{c}\text { Média } \\
\text { quadrática }\end{array}$ \\
\hline Regressão & $5,96.10^{10}$ & 4 & $1,49.10^{10}$ \\
Resíduo & $4,02.10^{8}$ & 9 & $4,50.10^{7}$ \\
Falta de ajuste & $3,09.10^{8}$ & 4 & $7,70.10^{7}$ \\
Erro puro & $9,30.10^{7}$ & 5 & $1,90.10^{7}$ \\
Total & $6,00.10^{10}$ & 13 & \\
\hline
\end{tabular}

\% de variação explicada: 99,33

\% máxima de variação explicável: 99,84

Para o modelo proposto, o termo $\left(\mathrm{x}_{2}\right)^{2}$ da equação foi desprezado, pois o mesmo não foi significativo para a determinação de $y$.

$$
\begin{aligned}
& y=36983+72604 x_{1}+17127 x_{2}+42781\left(x_{1}\right)^{2}+19392 x_{1} x_{2} \\
& \begin{array}{llll}
( \pm 2277) & ( \pm 2370) & ( \pm 2370) & ( \pm 2460)
\end{array}
\end{aligned}
$$

Quanto às fibras avaliadas, a PDMS/DVB foi escolhida como a de melhor rendimento, embora a fibra PA tenha mostrado um sutil aumento na área do pico cromatográfico, para o carbaril (Figura 3). Os motivos pelos quais a fibra PDMS/DVB foi escolhida está relacionado aos melhores resultados na precisão das duplicatas para cada ponto, assim como sua característica de polaridade mista, podendo esta fibra interagir tanto com compostos de baixa polaridade como o padrão substituto pirimicarbe, o analito carbaril e compostos de maior polaridade como o 1-naftol produto de degradação térmica do carbaril. ${ }^{40,41}$

Após a escolha da fibra, observou-se que o comportamento não linear da superfície de resposta sugere uma expansão do planejamento linear para quadrático, o que pode ser confirmado pelo gráfico de superfície de resposta após expansão (Figura 4) e pelo estudo da ANOVA análise de variância (Tabela 2).

De acordo com o gráfico da Figura 4, a variável temperatura demonstra possuir bastante influência na extração do analito, confirmado pela Figura 5. Isso de fato é esperado para compostos com baixa constante de Henry na MEFS-headspace, o que indica que um

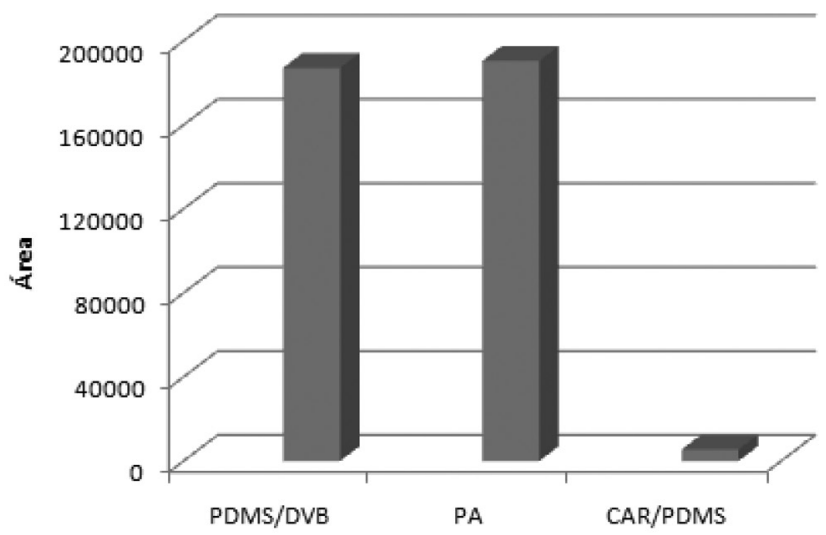

Figura 3. Rendimento das diferentes fibras na extração de carbaril

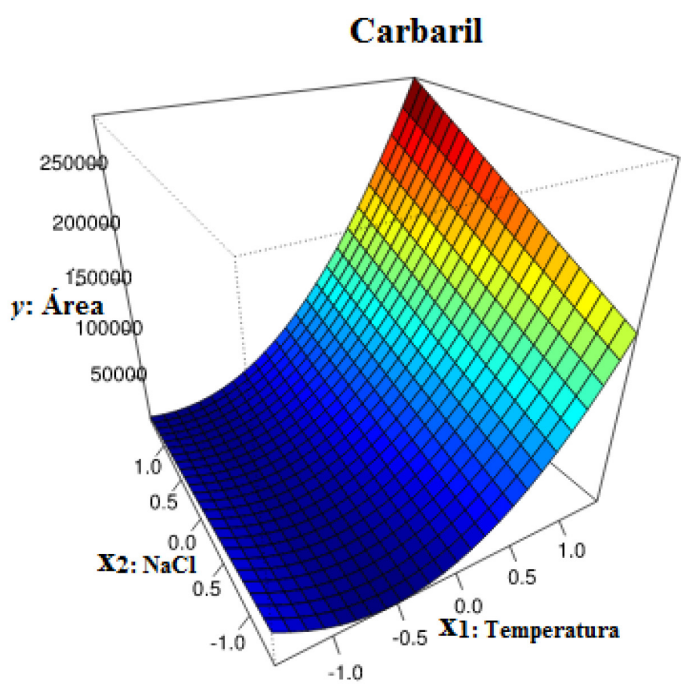

Figura 4. Gráfico de superfície de resposta na determinação da máxima extração de carbaril, por MEFS-headspace em função da temperatura $e$ força iônica $(\mathrm{NaCl})$

novo planejamento com a expansão da temperatura poderia melhorar os resultados, uma vez que a condição ótima para o planejamento parece não ter sido alcançada. Contudo, aumentar ainda mais a temperatura nessas condições significa também o aumento da pressão interna no interior do vial, o que não parece ser sensato por medidas de segurança, sendo que também ocorre o aumento do coeficiente de difusão no sistema, gerando uma diminuição da quantidade extraída quando o equilíbrio é alcançado, Portanto, é importante otimizar cuidadosamente a temperatura de extração para os tempos de equilíbrio mais curtos e para sensibilidades aceitáveis. ${ }^{42}$

Conforme observado no gráfico de pareto (Figura 5), a temperatura se mostrou como o único parâmetro significativo na extração do carbaril. A interpretação desse gráfico é importante quando se deseja realizar robustez do método de extração, uma vez que a pouca significância da força iônica e da interação entre as variáveis indicam que são possíveis variações na força iônica dentro do intervalo estudado, sem que influencie significativamente na eficiência da extração.

O estudo do tempo de equilíbrio (Figura 6) nos mostrou que a partir de 40 minutos houve uma pequena variação na área do pico cromatográfico do composto extraído, entrando em equilíbrio após 120 minutos. Para tempos de equilíbrio longos, um pré-equilíbrio para quantificação pode ser considerado, desde que as condições do sistema (temperatura, agitação, força iônica, $\mathrm{pH}$, dentre outros) sejam mantidas constantes. . $^{42,43}$ 


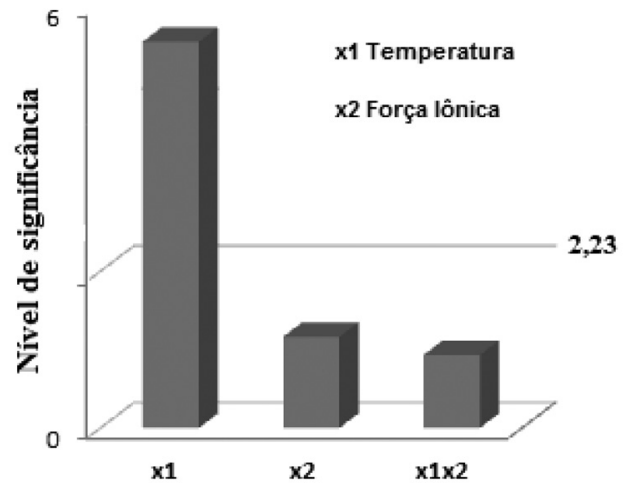

Figura 5. Gráfico de pareto, indicando quais parâmetros $(x 1, x 2)$ ou interação (x1x2), possuem influência significativa (nível de confiança de 95\%) sobre cada variável resposta

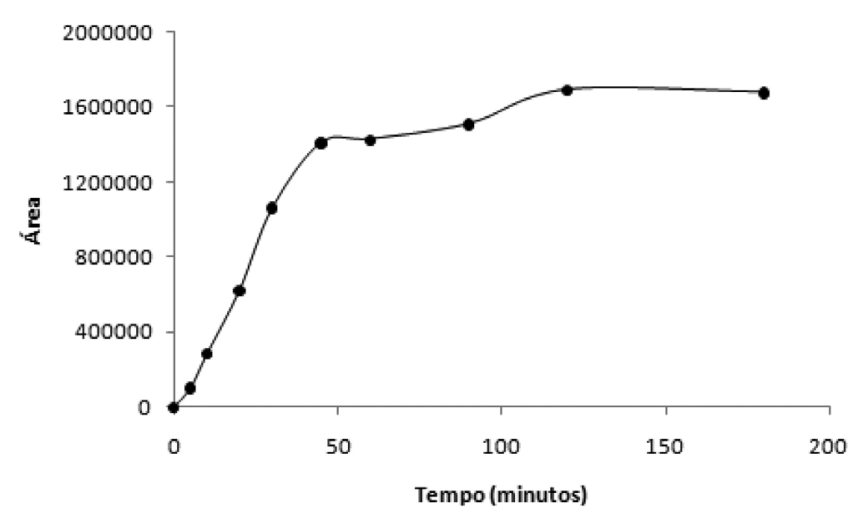

Figura 6. Gráfico de equilíbrio de extração em função do tempo

Por fim, dadas as condições de limitação no aumento da temperatura, discutidas anteriormente, e das características do analito e do modo de extração (headspace), e devido ao baixo valor na constante de Henry do composto $\left(9,20.10^{-5} \mathrm{~Pa} \mathrm{~m}^{3} \mathrm{~mol}^{-1}\right),{ }^{41}$ foi adotado um tempo de extração (pré-equilíbrio) para o método de 40 minutos. Esse tempo é necessário pelo fato de o analito precisar difundir-se a partir da fase condensada antes de atingir a fibra. Nesse caso, a extração pode ser otimizada com agitação mais eficiente ou, o que seria inviável, o aumento da temperatura de extração. ${ }^{42}$ Com isso, observa-se que incorporar as variáveis agitação e tempo de extração, em um novo planejamento experimental, possam otimizar ainda mais o tempo de extração, tornando o método ainda mais eficiente.

\section{Degradação térmica do carbaril na MEFS-headspace determinada por $\mathbf{R M N ~ H ^ { 1 }}$}

Como pode ser observado na Tabela 3, os deslocamentos referentes aos padrões individuais do carbaril e do 1-naftol, o singleto referente ao H-2' em 2,85 ppm é específico para o carbaril (Figura 7), sendo que a relação entre esse singleto e o duplo dubleto em 8,21 ppm no H-8 do 1-naftol pode fornecer de maneira direta a relação de degradação térmica do carbaril em 1-naftol nas condições de extração estudada, que seria de 7,75\% de 1-naftol produzido. ${ }^{8}$

A Figura 7 mostra o espectro $\mathrm{RMN} \mathrm{H}^{1}$ do padrão carbaril após este ser submetido às condições de MEFS-headspace com expansão dos sinais 6,6-8,2 ppm. Como pode ser observado, a determinação de degradação pôde ser determinada pela relação entre os sinais de hidrogênio aromático H-8 do carbaril e H-8 do 1-naftol, por estarem em um ambiente químico muito semelhante, sendo o sinal H-8 do carbaril um multipleto em 7,94 ppm e do H-8 do 1-naftol um duplo dubleto em 8,21 ppm. Assim, ao considerar o sinal do H-8 do carbaril como 1,00, obtém-se um sinal de integração correspondente para o H-8 do 1-naftol de 0,08, sugerindo que aproximadamente $8,0 \%$ de 1-naftol é produzido pela degradação térmica do carbaril após ser submetido as condições de MEFS do método, resultado esse semelhante à relação anterior feita entre os sinais H-8 do 1-naftol e H-2' do carbaril.

A análise de $\mathrm{RMN} \mathrm{H} \mathrm{H}^{1}$ mostra, com essa pequena quantidade de carbaril convertida em 1-naftol, que o processo de hidrólise foi pouco significativo; sabemos que esse processo pode ser expressivo em pHs neutro e básico, ${ }^{44}$ assim como por influência do solvente e em temperaturas elevadas. ${ }^{8}$ Por esse motivo adotou-se o pH 5,5 e optou-se por não aumentar a temperatura de extração, expandindo com isso o planejamento experimental e, nessas condições, observar a interação do próprio analito com os revestimentos das fibras de MEFS, principalmente por esse trabalho não avaliar a metabolização (hidrólise) do carbaril no organismo humano e por não saber em qual forma predominante o composto se encontraria no plasma.

\section{Validação do método}

Seletividade

Pelo fato da matriz biológica "plasma" ser uma matriz bastante complexa, é importante que se consiga uma boa seletividade para o método. A utilização da MEFS-HS como técnica de preparo da amostra se mostra bastante interessante, juntamente com o uso do modo MIS (Monitoramento de Íons Selecionado) no EM, monitorando

Tabela 3. Dados de $\mathrm{RMN}{ }^{1} \mathrm{H}$ determinados pelos padrões do carbaril e 1-naftol (Metanol deuterado/500 MHz)

\begin{tabular}{|c|c|c|c|c|c|c|c|}
\hline \multicolumn{4}{|c|}{ Carbaril } & \multicolumn{4}{|c|}{ 1-naftol } \\
\hline $\mathrm{H}$ & $\delta^{1} \mathrm{H}(\mathrm{ppm})$ & Multp. & $J(\mathrm{~Hz})$ & $\mathrm{H}$ & $\delta^{1} \mathrm{H}(\mathrm{ppm})$ & Multp. & $J(\mathrm{~Hz})$ \\
\hline 1 & - & - & - & 1 & - & - & - \\
\hline 2 & 7,24 & $\mathrm{~d}$ & 7,45 & 2 & 6,83 & dd & $7,35-0,90$ \\
\hline 3 & 7,46 & $\mathrm{t}$ & 7,88 & 3 & 7,25 & $\mathrm{t}$ & 4,05 \\
\hline 4 & 7,74 & $\mathrm{~d}$ & 8,25 & 4 & 7,32 & $\mathrm{~d}$ & 8,25 \\
\hline $4 a$ & - & - & - & $4 a$ & - & - & - \\
\hline 5 & 7,89 & $\mathrm{~m}$ & - & 5 & 7,75 & dd & $6,75-2,35$ \\
\hline 6 & 7,51 & $\mathrm{~m}$ & - & 6 & 7,39 & $\mathrm{~m}$ & - \\
\hline 7 & 7,52 & $\mathrm{~m}$ & - & 7 & 7,42 & $\mathrm{~m}$ & - \\
\hline 8 & 7,94 & $\mathrm{~m}$ & - & 8 & 8,21 & dd & $8,18-1,28$ \\
\hline $8 \mathrm{a}$ & - & - & - & $8 \mathrm{a}$ & - & - & - \\
\hline $1^{\prime}$ & - & - & - & & & & \\
\hline 2 ' & 2,85 & $\mathrm{~s}$ & - & & & & \\
\hline
\end{tabular}




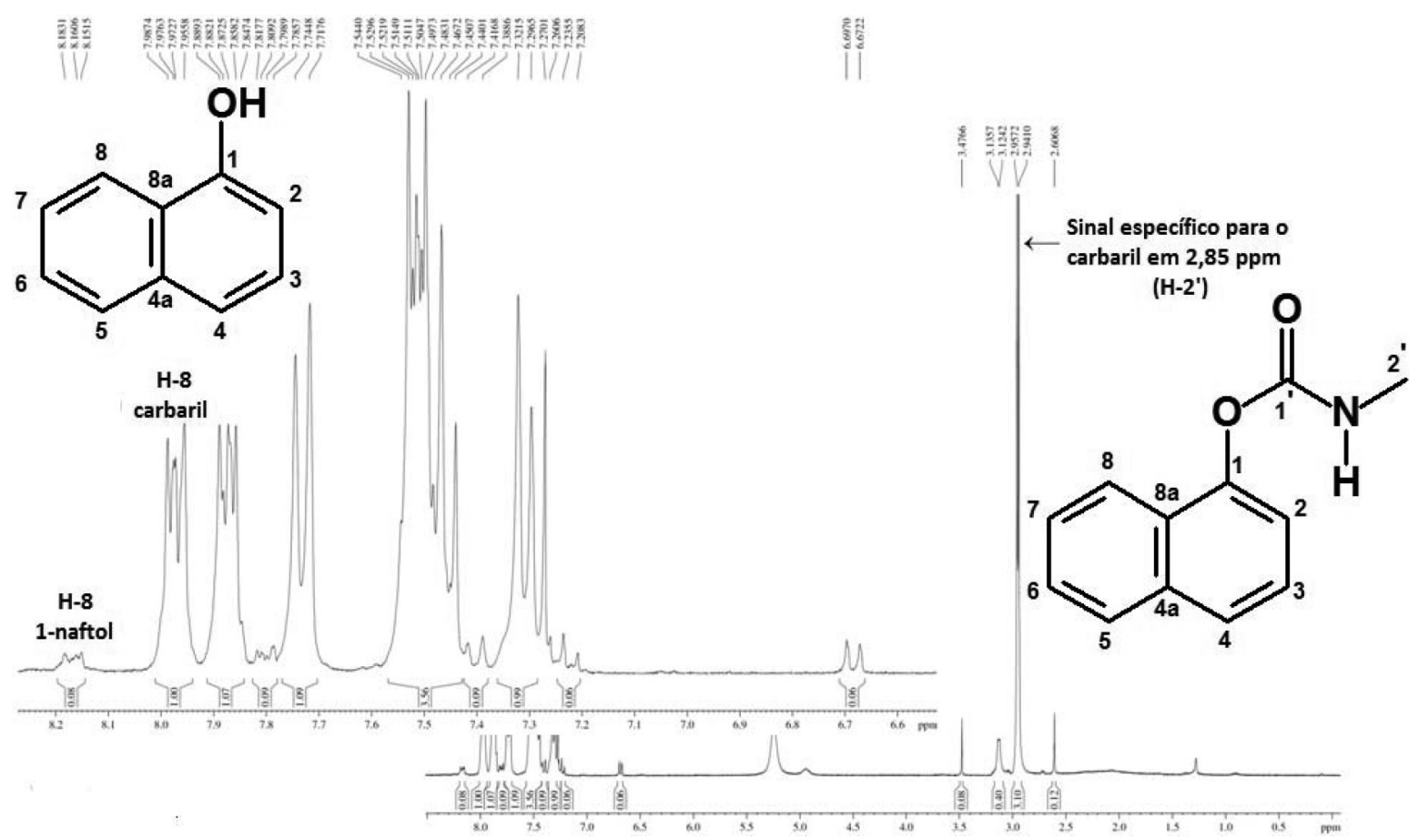

Figura 7. Espectro RMN $H^{l}$ do padrão carbaril após ser submetido às condições de MEFS-headspace com expansão dos sinais 6,6 - 8,2 ppm

apenas o íon de interesse e eliminando assim possíveis interferentes.

O método se mostrou seletivo para as amostras, uma vez que não foram detectados interferentes nos mesmos tempos de retenção dos compostos analisados 1-naftol e pirimicarbe (PS), com as injeções dos brancos de 6 indivíduos, sendo 4 dessas normais, uma lipêmica e outra hemolisada; isso garante que o método pode ser utilizado com confiabilidade para amostras de plasma de pacientes com alguma dessas características.

\section{Efeito residual}

A avaliação do efeito residual é importante para garantir que uma análise não seja afetada por análises anteriores. Foi observado um resíduo inferior a $20 \%$ da resposta do analito nas amostras processadas do LIQ e inferiores a $5 \%$ da resposta do padrão substituto. O baixo efeito residual já era esperado, uma vez que uma limpeza da fibra era realizada após cada injeção da amostra. A avaliação desse efeito residual serve como indicativo de que as etapas de limpeza realizadas foram bem-sucedidas.
Linearidade, Limite de Detecção $(L D)$ e Limite de Quantificação $(L Q)$

No estudo de linearidade do método e escolha do modelo matemático foram realizados testes estatísticos de heterocedasticidade, ${ }^{31-33}$ que mostraram um alto valor de significância da regressão e uma baixa falta de ajuste para o modelo com $95 \%$ de confiança, segundo ANOVA, assim como uma melhora considerável no valor do coeficiente de correlação $r_{w i} 0,999$ (Tabela 4).

O MMQP (Método dos Mínimos Quadrados Ponderados) também forneceu menores valores de LD e LQ comparado ao MMQO (Método dos Mínimos Quadrados Ordinários) ou método tradicional de regressão, o que determina um modelo mais sensível e realístico do conjunto de dados. Embora os valores de LD e LQ desse estudo sejam mais elevados que os encontrados por Petropelou et al.,$^{10} \mathrm{em}$ que o LD e o LQ foram respectivamente, 0,03 e $0,11 \mathrm{ng} \mathrm{mL}^{-1}$, utilizando um CG-EM/EM, ele nos proporciona uma boa sensibilidade, principalmente por avaliar a presença de carbaril pelo sinal de seu produto de degradação térmica 1-naftol. O valor de LD obtido neste

Tabela 4. Figuras de mérito da validação do método

Equação da reta MMQP* $(n=6 \times 3=18)$

Desvio padrão de intercessão $\left(b_{w i}\right)$

Desvio padrão de inclinação da reta $\left(a_{w i}\right)$

Coeficiente de correlação $\left(r_{w i}\right)$

Limite de Detecção (ng $\left.\mathrm{mL}^{-1}\right)$

Limite de Quantificação (ng $\mathrm{mL}^{-1}$ )

\begin{tabular}{|c|c|c|c|c|}
\hline \multirow{2}{*}{ Níveis de concentração (ng mL $\left.\mathrm{m}^{-1}\right)$} & \multicolumn{2}{|c|}{ Repetibilidade CV\% $(\mathrm{n}=5)$} & \multicolumn{2}{|c|}{ Recuperação \% $(\mathrm{n}=5)$} \\
\hline & Intra-corrida & Inter-corrida & Intra-corrida & Inter-corrida \\
\hline Baixo $(12,0)$ & 10,01 & 13,41 & 116,21 & 118,25 \\
\hline Médio $(90,0)$ & 8,15 & 10,01 & 95,13 & 104,74 \\
\hline Alto $(180,0)$ & 9,34 & 12,73 & 108,60 & 112,41 \\
\hline
\end{tabular}

$y=0,001249 x+0,003770$

$6,21 \times 10^{-4}$

$1,87 \times 10^{-5}$

0,999

1,5

*MMQP (Método dos Mínimos Quadrados Ponderados). 
trabalho, no entanto, foi menor do que o validado por Barr et al.,$^{45}$ que obtiveram $20 \mathrm{ng} \mathrm{mL}^{-1}$ utilizando CG-EM.

\section{Precisão e exatidão}

A precisão e exatidão do método (Tabela 4) foram determinadas pela repetibilidade e recuperação intra e inter-corrida. As variações máximas são de $20 \%$ para o LIQ e de $15 \%$ para as demais concentrações; nesse estudo, as maiores variações para CV\% foram de $13,41 \%$ para o LIQ e de $12,73 \%$ para as demais concentrações e variações máximas de recuperação 18,25\% para o LIQ e 12,41\% para as demais concentrações.

\section{Aplicação do método em amostras de pacientes voluntários}

Nenhuma amostra apresentou concentração do composto 1-naftol acima do limite de detecção do método aqui proposto e essa não detecção pode ter ocorrido devido à rápida metabolização do carbaril, seguida por sua eliminação através do sistema excretor, características essas não estudadas nesse trabalho. Sugere-se, então, que sejam realizados testes que observem o comportamento do analito no organismo, ou que as coletas, para esse tipo de análises, ocorram ao final do expediente e ainda no ambiente de trabalho, na tentativa de evitar possíveis processos metabólicos e eliminação do analito. Outro fator que pode ter contribuído bastante foi o fato de grande parte de trabalhadores que lidam diretamente com a aplicação dos agrotóxicos não comparecerem à coleta de sangue, podendo essa ausência estar associada aos intensos conflitos registrados na região de Limoeiro do Norte-CE. ${ }^{46-48}$

\section{CONCLUSÃO}

As condições para a análise de carbaril no plasma humano utilizando MEFS-headspace preservam a fibra, pelo fato de não ocorrer o contato direto entre a fibra e material biológico, tornando o procedimento de preparação da amostra mais simples, com apenas $0,5 \mathrm{~mL}$ de amostra de plasma utilizado, demandando menores quantidades de solventes orgânicos, com menos custos associados e baixo impacto ambiental. $\mathrm{O}$ método proposto alcançou baixos limites de detecção e quantificação, assim como boa precisão e exatidão, principalmente com a aplicação do Método dos Mínimos Quadrados Ponderados. Portanto, o método proposto pode ser considerado como uma boa alternativa no monitoramento de carbaril em plasma sanguíneo, pelo sinal analítico do 1-naftol.

\section{REFERÊNCIAS}

1. http://unesdoc.unesco.org/images/0023/002318/231823E.pdf, acessada em fevereiro 2017.

2. http://www.iea.sp.gov.br/out/verTexto.php?codTexto=13679, acessada em fevereiro de 2017.

3. Pinto, M. I.; Sontag, G.; Bernardino R. J.; Noronha, J. P.; Microchem. J. 2010, 96, 225.

4. Fernandes, V. C.; Domingues, V. F.; Mateus, N.; Delerue-Matos, C.; J. Agric. Food Chem. 2011, 59, 7582.

5. Jin, B.; Xie, L.; Guo, Y.; Pang, G; Food Res. Int. 2012, 46, 399.

6. Gomez-Ramos, M. M.; Ferrer, C.; Malato, O.; Aguera, A.; Fernandez-Alba, A. R.; J. Chromatogr. A 2013, 1287, 24.

7. http://www.abrasco.org.br/site/wp-content/uploads/2015/03/Dossie_ Abrasco_01.pdf, acessada em fevereiro de 2017.

8. Przybylski, C.; Bonnet, V.; J. Chromatogr. A 2009, 1216, 4787.

9. Yang, E-Y.; Shin, H-S.; J. Chromatogr. A 2013, 1305, 328.

10. Petropoulou, S-S. E.; Tsarbopoulos, A.; Siskos, A. S.; Anal. Bioanal. Chem. 2006, 385, 1444.
11. Chu, N.; Fan, S.; Spectrochim. Acta, Part A 2009, 74, 1173.

12. Padilla, S.; Marshall, R. S.; Hunter, D. L.; Lowit, A.; Toxicol. Appl. Pharmacol. 2007, 219, 202.

13. Herr, D. W.; Mwanzab, J-C.; Lykea, D. F.; Graffc, J. E.; Mosera, V. C.; Padilla, S.; Toxicology 2010, 276, 172.

14. Eraslan, G.; Kanbur, M.; Silici, S.; Food Chem. Toxicol. 2009, 47, 86.

15. Moreno, A. J. M.; Serafim, T. L.; Oliveira, P. J.; Madeira, V. M. C.; Chemosphere 2007, 66, 404.

16. Jorsaraei, S. G. A.; Maliji, G.; Azadmehr, A.; Moghadamnia, A. A.; Faraji, A. A.; Environ. Toxicol. Pharmacol. 2014, 38, 838.

17. Lin, C. C.; Hui, M. N. Y.; Cheng, S. H.; Toxicol. Appl. Pharmacol. 2007, $222,159$.

18. Matos, P.; Fontaínhas-Fernandes, A.; Peixoto, F.; Carrola, J.; Rocha, E.; Pestic. Biochem. Physiol. 2007, 89, 73.

19. Boone, M. D.; Hammond, S. A.; Veldhoen, N.; Youngquist, M.; Helbing, C. C.; Aquat. Toxicol. 2013, 130-131, 139.

20. Lima, M. P.; Cardoso, D. N.; Soares, A. M.; Loureiro S.; Ecotoxicology and Environ. Saf. 2015, 114, 263.

21. Aprea, C.; Colosio, C.; Mammone, T.; Minoia, C.; Maroni, M.; J. Chromatogr. B 2002, 769, 191.

22. Mostafa, A.; Medley, G.; Roberts, D. M.; Mohamede, M. S.; Elshanawanif, A. A.; Roberts, M. S.; Liua, X.; J. Chromatogr. B 2011, 879, 2234.

23. Araoud, M.; Gazzah, N.; Douki, W.; Najjar, M. F.; Kenani, A.; Afr. J. Biotechnol. 2012, 11, 12579.

24. Tennakoon, D. A. S. S.; Karunarathna, W. D. V.; Udugampala, U. S. S.; Forensic Sci. Int. 2013, 227, 106.

25. Wang, H-S.; Chen, Z-J.; Wei, W.; Man, Y-B; Giesy, J. P.; Du, J.; Zhang, G.; Wong, C. K-C.; Wong, M-H.; Environ. Int. 2013, 54, 18.

26. Ribeiro, H. L. J.; Oliveira, R. T. G.; Maia, A. R. S.; Filho, L. I. P. F.; de Sousa, J. C.; Heredia, F. F.; Magalhães, S. M. M.; Pinheiro, R. F.; Hematol. Oncol. 2015, 33, 220.

27. Filho, L. I. P. F.; Dissertação de Mestrado, Universidade Federal do Ceará, Brasil, 2013.

28. Agência Nacional de Vigilância Sanitária (ANVISA); Resolução RE $n^{o}$ 899, de 29/05/2003.

29. Agência Nacional de Vigilância Sanitária (ANVISA); Resolução RE $n^{o}$ 27, de 17/05/2012.

30. Ribani, M.; Bottoli, C. B. G.; Collins, C. H.; Jardim, I. C. S. F.; Quim. Nova 2004, 27, 5, 771.

31. Neto, B. B.; Scarminio, I. S.; Bruns, R. E. Como fazer experimentos, $4^{\text {a }}$ ed., Bookman: Porto Alegre 2010.

32. Miller, J. N.; Miller, J. C.; Statistics and Chemometrics for Analytical Chemistry, $5^{\mathrm{a}}$ ed., Pearson Prentice Hall: Harlow, 2005.

33. Ministerio da Agricultura, Pecuária e Abastecimento (MAPA), Manual de Garantia da Qualidade Analítica: Brasília, 2011.

34. Souza, F. T. C; Barbosa, P. G. A.; Camelo, A. L. M.; Longhinot, E.; Becker, H.; Trabalho não publicado.

35. Przybylski, C.; Hommet, F.; J. Chromatogr. A 2008, 1201, 78.

36. Raeppel, C.; Niefa, M.; Fabritius, M.; Racaulta, L.; Appenzellerb, B. M.; Milleta, M.; J. Chromatogr. A 2011, 1218, 8123.

37. Grob, R. L.; Barry, E. F.; Modern Practice of Gas Chromatography, $4^{\mathrm{a}}$ ed., Wiley-Interscience: New Jersey, 2004.

38. Pawliszyn, J.; Solid Phase Microextraction: Theory and Practice. WileyVHC: New York, 1997.

39. Theodoridis, G.; Kpster, E. H. M.; Jong, G. J.; J. Chromatogr. B 2000, $745,49$.

40. Pereira, F. V.; Cardeal, Z. L.; Quim. Nova 2005, 28, 646.

41. http://sitem.herts.ac.uk/aeru/iupac/atoz.htm\#P, acessada em fevereiro de 2017.

42. Pawliszyn, J.; Chromatogr. Sci. 2000, 38, 270.

43. Zhang, J.; Lee, H. K.; J. Chromatogr. A 2006, 1117, 31.

44. Ballesteros, E.; Gallego, M.; Valcircel, M. J. Chromatogr. 1993, 633, 169. 
45. Barr, D. B.; Barr, J. R.; Maggio, V. L.; Whitehead Jr., R. D.; Sadowski, M. A.; Whyatt, R. M.; Needham, L. L.; J. Chromatogr. B 2002, 778, 99.

46. http://www.ecodebate.com.br/2010/05/13/limoeiro-do-norte-ceaudiencia-debate-agrotoxico-e-caderno-conflitos-no-campo-2009/, acessada em fevereiro de 2017.
47. http://diariodonordeste.verdesmares.com.br/cadernos/regional/ agrotoxico-esta-com-maior-poder-de-contaminacao-1.285352, acessada em fevereiro de 2017.

48. http://terradedireitos.org.br/2014/03/25/artigo-trabalho-agrotoxicos-emorte-no-campo-uma-longa-espera-por-justica/, acessada em fevereiro de 2017. 